CASE REPORT

\title{
Reattachment of Complicated Crown: Root Fracture: A Case Report
}

\author{
Amal Devadas ${ }^{1}$, Praveena Geetha ${ }^{2}$, K Radhakrishnan Nair ${ }^{3}$, Deepak Joby ${ }^{4}$, Sreelekshmy Chandrababu ${ }^{5}$
}

\begin{abstract}
Aim and background: Complicated crown-root fractures constitute a major share of all dental injuries, most commonly affecting the maxillary anterior teeth. The main objective while treating complicated crown fracture involves pain management and immediate restoration of function and esthetics. When the fractured segments are closely approximating, root canal treatment followed by reattachment of the fractured segment with fiber post reinforcement is a suitable option. This technique provides excellent esthetics as it maintains the original contacts and contour, translucency, alignment, surface texture, and position of the teeth.

Case description: A 27-year-old male reported to the clinic immediately following a road traffic accident. Clinical and radiographic examination revealed a complicated oblique fracture that was extending subgingivally in the palatal aspect in relation to 21 and 22 . It was decided to perform a single visit root canal treatment followed by reattachment of the fractured segment using fiber-reinforced post. A midline diastema was noted following the attachment of the fractured segment which was corrected using direct composite resin restoration.

Conclusion: The presented case demonstrates conservative management of complicated crown root fracture involving 21 and 22 by reattachment using fiber-reinforced post followed by midline diastema correction using composite resin.

Keywords: Anterior teeth, Composite resin, Crown-root fracture, Fiber-reinforced post, Reattachment, Trauma.

Conservative Dentistry and Endodontic Journal (2021): 10.5005/jp-journals-10048-0070
\end{abstract}

\section{INTRODUCTION}

Complicated crown fractures are one of the most common types of fractures involving anterior teeth. Most commonly affecting the maxillary incisors, forming $96 \%$ of all crown fractures. ${ }^{1}$ Etiological factors of crown-root fractures of permanent dentition are falls, sports injuries, car accidents, and foreign body striking the teeth. ${ }^{2}$ Such a tooth requires immediate clinical attention.

The ultimate goal of any dental emergency treatment is to re-establish normal tooth position and function. Numerous techniques to restore fractured crowns have emerged in recent years. Conventionally, composite restorations and post-and-core supported prostheses are the most commonly used modalities. The treatment plan may vary when the fractured fragment is available and intact.

Reattachment of the fractured fragment using the adhesive technique can be the most immediate and conservative treatment option concerning esthetics if the fractured portion is intact and the margins are preserved as it involves minimal intervention and biological restoration of the natural tooth. The tooth reattachment technique can restore a patient's esthetics, form, contour, alignment, translucence, surface texture, and position of the tooth within a short span of time. Many options are there for the reattachment of the fractured tooth fragment. In case of complicated crown-root fractures where the fractured segments are closely approximating, root canal treatment followed by reattachment of the fractured segment with fiber post reinforcement is a suitable option. Toothcolored fiber posts have several advantages such as esthetics, bonding to the tooth structure, and a similar modulus of elasticity as that of dentin. Also, it provides a monoblock effect thereby increasing the retention of the complex by enhancing the fitness of the post for the prepared root canal. ${ }^{3}$

The presence of midline diastema is often considered an esthetic problem by patients. The most common factors in the
${ }^{1-5}$ Department of Conservative Dentistry and Endodontics, Azeezia College of Dental Sciences and Research, Kollam, Kerala, India

Corresponding Author: Amal Devadas, Department of Conservative Dentistry and Endodontics, Azeezia College of Dental Sciences and Research, Kollam, Kerala, India, Phone: +91 8073062579, e-mail: dramal.devadas@gmail.com

How to cite this article: Devadas A, Geetha P, Radhakrishnan Nair K, et al. Reattachment of Complicated Crown: Root Fracture: A Case Report. Cons Dent Endod J 2021;6(1):18-21.

Source of support: Nil

Conflict of interest: None

development of diastema are tooth size discrepancies, an excessive vertical overlap of incisors, mesiodistal angulations, generalized spacing, labiolingual incisor inclination, frenum attachment, and pathological conditions. Diastemas can be treated in a multitude of ways including orthodontic closure, restorative therapy, surgical correction, or multidisciplinary approach depending upon the particular case and etiology of diastema. Increased patient demand for the minimally invasive esthetic procedure and the improved physical properties of current composites has resulted in the extensive utilization of direct bonding of composite resin to anterior teeth.

In this case report, we are discussing the management of a complicated crown fracture involving a central and lateral incisor by reattachment using fiber post followed by a midline diastema correction using direct composite resin.

\section{Case Description}

A 27-year-old male was referred from the department of oral and maxillofacial surgery with a detached fragment of 21 and 22 


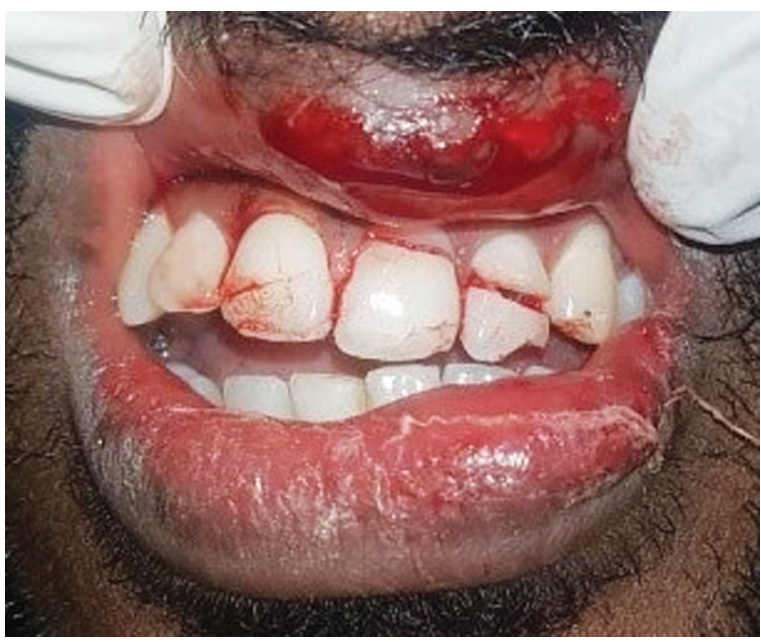

Fig. 1: Preoperative clinical view-labial

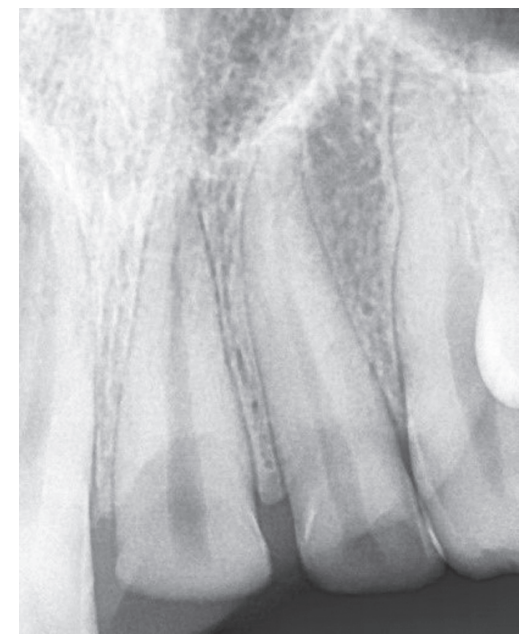

Fig. 3: Preoperative radiograph

immediately following a road traffic accident. A lacerated wound was present in the left upper labial mucosa which was sutured. He was in pain due to pulp exposure. Clinical and radiographic examination revealed a complicated oblique fracture that was extending subgingivally in the palatal aspect. Periodontal ligament space was intact and no root fracture was seen in relation to 21 and 22 (Figs 1 to 3 ).

Medical history was non-contributory. After discussing various treatment options with the patient, single visit root canal treatment was started. The fractured segment was immediately stored in artificial saliva (ICPA, India). It was decided to use a fiber post for reattaching the fractured fragment. The patient's informed consent was obtained before the treatment. Local anesthesia was administered (1.0 cc of lidocaine $2 \%$ with 1:80,000 epinephrine) and an access opening was done holding the fragments in position in respect to the related tooth. Access opening was done on the tooth fragments for easy adaption of the fiber post (Fig. 4). Following root canal treatment in relation to 21 and 22 (Fig. 5), laser gingivectomy was performed since the lingual margins were located subgingivally in relation to 21,22 and also to attain complete hemostasis. Post space was prepared using peeso reamers of sizes 1, 2, and 3. A fiberreinforced post of size 2 (Angelus, REFORPOST, Londrina, Brazil) was selected and the length of the post was adjusted so that $2 \mathrm{~mm}$ was

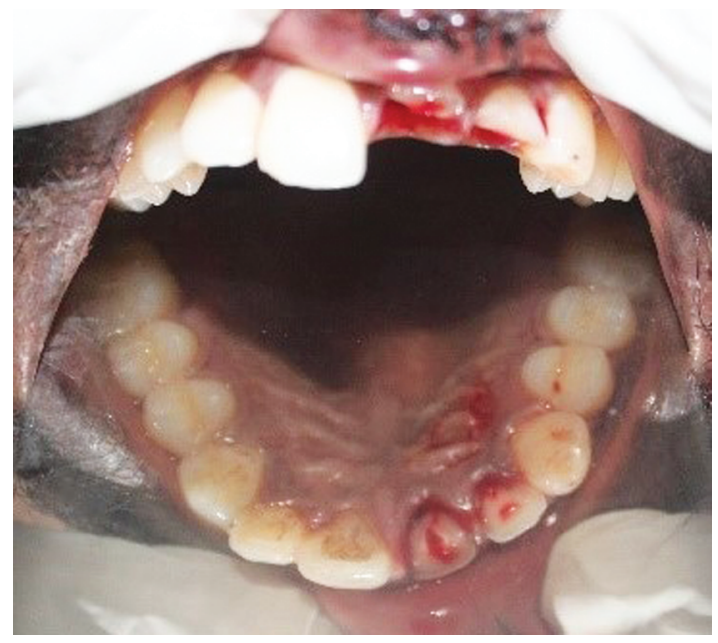

Fig. 2: Preoperative clinical view—palatal

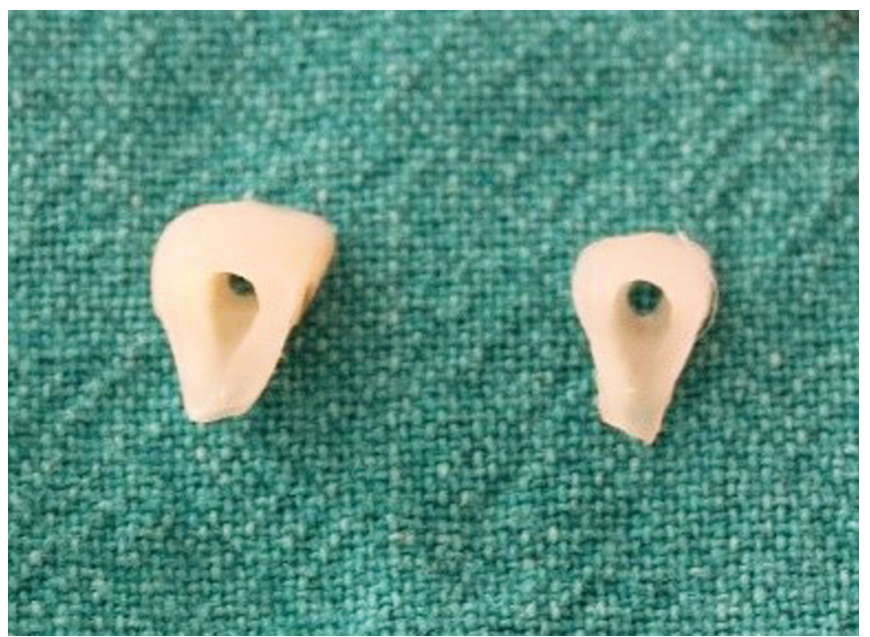

Fig. 4: Fractured segments

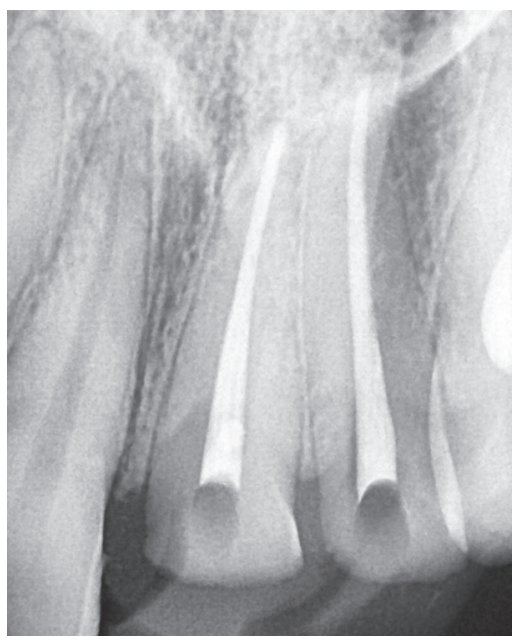

Fig. 5: Postobturation radiograph

extending coronally to the fractured segment. The prepared post space was conditioned and adhesive application was done on fiber post, post space, and tooth fragments. The dual-cure resin luting cement was injected into the post space and the fiber post. The fiber 


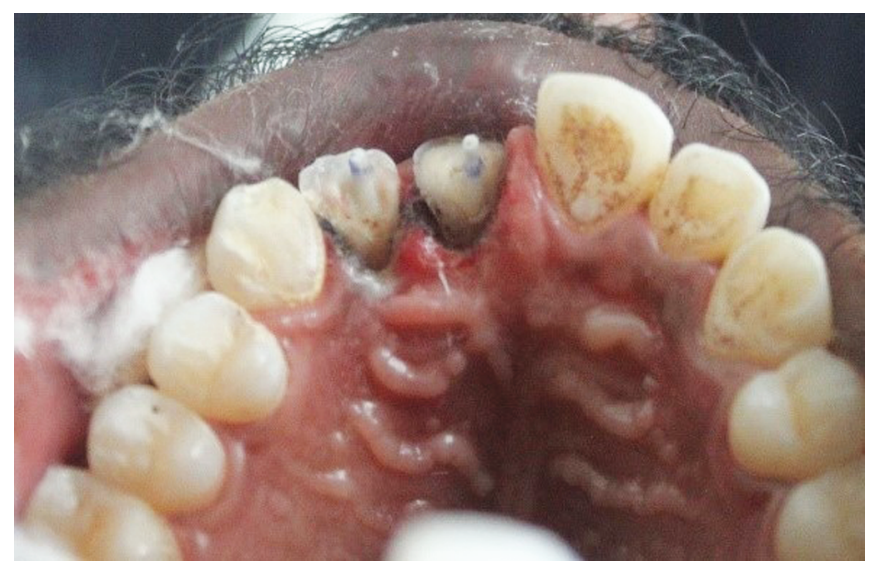

Fig. 6: Fiber post luted in 21 and 22

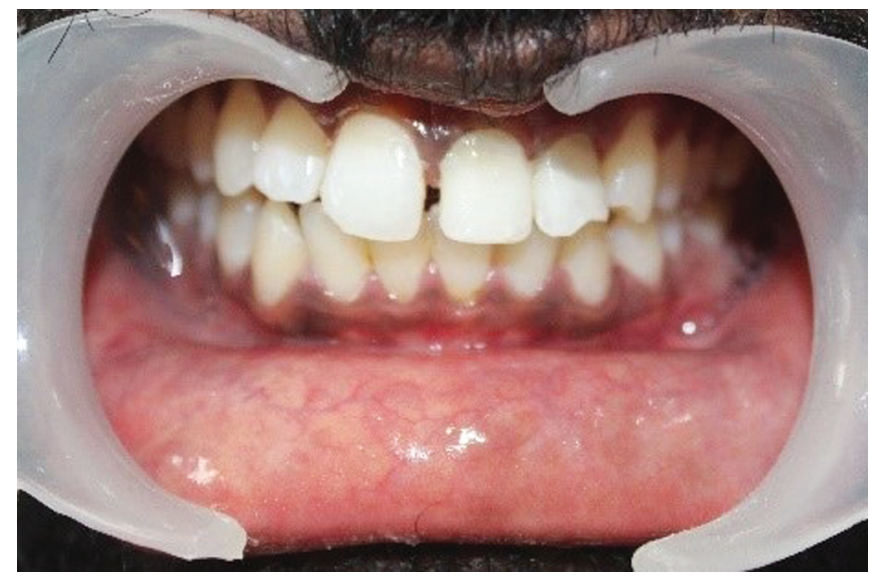

Fig. 8: Postoperative view

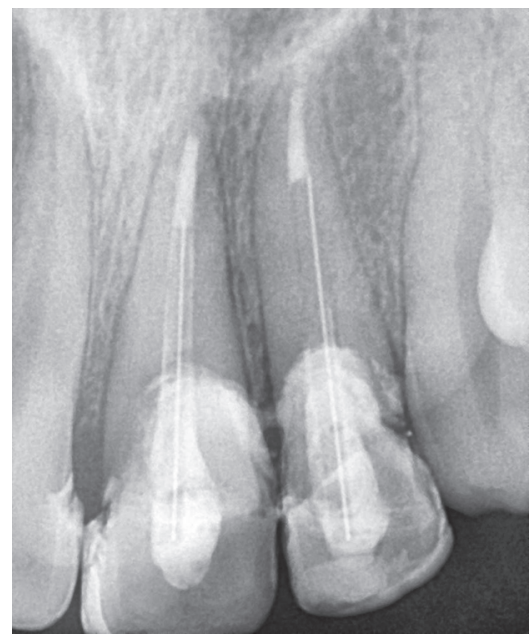

Fig. 10: 6 months follow-up radiograph

post was luted into the post space following which, light-curing was done (Fig. 6). The tooth fragments and tooth surface were etched and a bonding agent was applied. Tooth fragments were then attached using resin cement and were cured (Fig. 7). Midline diastema was noted following the attachment of fragments (Fig. 8). An esthetic correction was planned for the same using composite on the next recall.

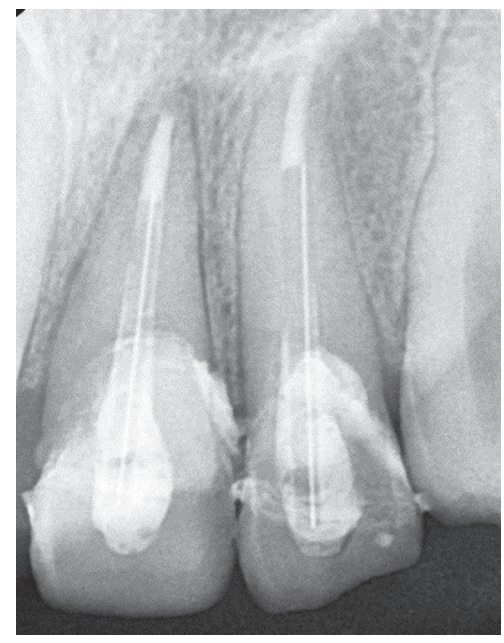

Fig. 7: Post reattachment radiograph

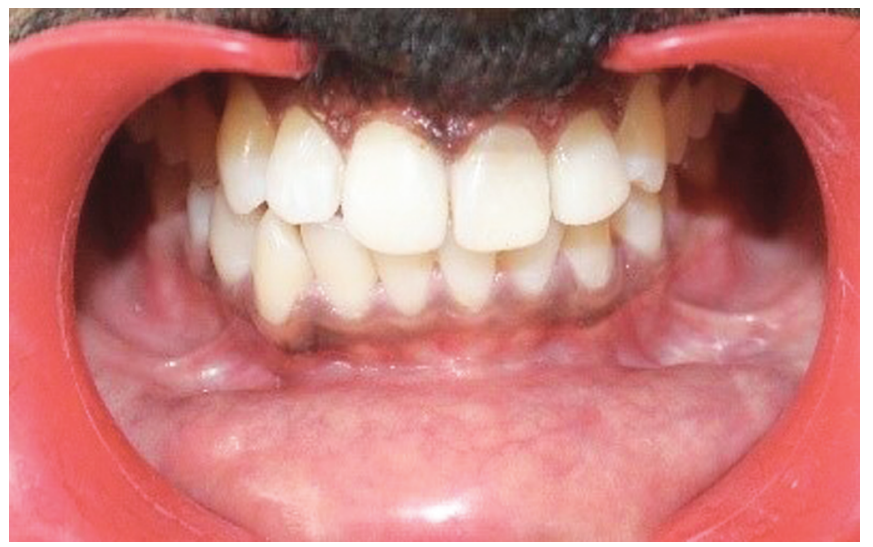

Fig. 9: Postoperative view after midline diastema correction

At 1-week recall midline diastema correction was done using composite resin matching appropriate shades (Fig. 9). The patient was further recalled after, 3 weeks, 3 months, and 6 months for evaluation and was asymptomatic. The patient was kept on periodic review and it was observed that both endodontic and restorative treatments remained clinically acceptable through each visit. The clinical and radiographic pictures after 6 months were satisfactory (Fig. 10).

\section{Discussion}

Restoring structural integrity, function, and esthetics are the most important factors when it comes to the management of traumatized anterior teeth. First described by Chosack and Eidelman, ${ }^{4}$ if the fractured fragment is available intact at the right time, reattachment has to be the most desired treatment. As it offers good esthetics, an exact recreation of contacts and contour, and a very cost-effective treatment alternative rather than restoring it prosthetically. It also aids in the social and psychological well-being of a patient. In addition, tooth fragment reattachment allows restoration of the tooth with minimal sacrifice of the remaining tooth structure.

Many case reports and researches in the literature suggest that reattachment of a fractured tooth fragment is a viable approach for the treatment of coronal fracture of anterior teeth when the fractured segment is available. ${ }^{5,6}$ The success of the reattachment 
depends on several factors: hydration of the fractured segment while outside the oral cavity is one of them. ${ }^{7}$ This is necessary to maintain the original esthetic appearance of the tooth and also ensures adequate bond strength. If the extraoral time of the fractured fragment increases, dehydration of the fragment can occur. Therefore, to prevent this, it is recommended that the fragment be kept in a medium such as physiologic saline, and the fragments were kept in artificial saliva to prevent dehydration.

When there is a substantial associated periodontal injury and/ or invasion of the biological width, the restorative management of the coronal fracture should also consider the rehabilitation of those associated tissues. In this case report, the fracture line extended subgingivally in the palatal area in relation to 21 and 22 but not violating the biological width. Hence, no osteotomy procedure was deemed necessary. Laser gingivectomy was preferred as it helps in the proper adhesion of the tooth fragments in a well-isolated field.

Reinforcement of the reattached fragments using posts has been widely reported in the literature., ${ }^{5,6}$ Although many techniques with various materials have been suggested, resinbased restorative materials with fiber post may be considered the best option because of several advantages such as a suitable elastic modulus, esthetics, good bonding between post and cement, lower chairside time, and minimal tissue removal. In addition to the preparation of the post space, bigger access was created in the coronal separated segment as leeway for the excess cement to flow out without build-up of any hydrostatic pressure. Reattached teeth are subjected to more stress and strain compared to the normal tooth. They are prone to re-fracture, ${ }^{8}$ so the reattached fragments have to be well bonded to withstand different types of forces. Modern composite materials with their advanced physical and esthetic properties can be used to create good quality esthetic restorations. In our case, direct bonding of composite resin to correct the midline diastema of reattached tooth aided to reinforce the tooth integrity as well as the longevity of the attached tooth fragment. Diastema closure was done using direct composite resin, thereby achieving a good esthetic and functional outcome.

\section{Conclusion}

The presented case demonstrates a conservative approach in restoring fractured anterior teeth in a simple, economical, effective alternative to restore the esthetic form and function of teeth.

\section{References}

1. Goenka P, Marwah N, Dutta S. Biological approach for management of anterior tooth trauma: triple case report. J Indian Soc Pedod Prev Dent 2010;28(3):223-229. DOI: 10.4103/0970-4388.73791.

2. Andreasen JO, Andreasen F, Andersson L. Textbook and color atlas of traumatic injuries to the teeth. 3rd ed., St Louis (MO): Mosby; 1994. pp. 314-334.

3. Tay FR, Pashley DH. Monoblocks in root canals: a hypothetical or a tangible goal. J Endod 2007;33(4):391-398. DOI: 10.1016/ j.joen.2006.10.009.

4. Chosack A, Eidelman E. Rehabilitating of a fractured incisor using patients natural crown: a case report. J Dentis Child 1994;71:19-21.

5. Iseri U, Ozkurt Z, Kazazoglu E. Clinical management of a fractured anterior tooth with reattachment technique: a case report with an 8-year follow up. Dent Traumatol 2011;27(5):399-403. DOI: 10.1111/j.1600-9657.2011.01015.x.

6. Kumari NBPS, Sujana V, Sunil CHR, et al. Reattachment of complicated tooth fracture: an alternative approach. Contemp Clin Dentis 2012;3(2):242-244. DOI: 10.4103/0976-237X.96843.

7. Shirani F, Sakhaei Manesh V, Malekipour MR. Preservation of coronal tooth fragments prior to reattachment. Aust Dent J 2013;58(3): 321-325. DOI: 10.1111/adj.12092.

8. Reis A, Loguercio AD, Kraul A, et al. Reattachment of fractured teeth: a review of literature regarding techniques and materials. Oper Dent 2004;29(2):226-233. 